\title{
NON-RIGID REGISTRATION ALGORITHM WITH SPATIALLY VARYING STIFFNESS PROPERTIES
}

\author{
Valerie Duay $^{1,2}$, Pierre-François D'Haese ${ }^{1}$, Rui Li $^{1}$, and Benoit M. Dawant ${ }^{1}$ \\ ${ }^{1}$ Department of Electrical Engineering and Computer Science, Vanderbilt University, Nashville, USA \\ ${ }^{2}$ Currently with the Signal Processing Institute, EPFL, Lausanne, Switzerland
}

\begin{abstract}
Non-rigid registration algorithms have been proposed over the years to register medical images to each other. One class of applications for these algorithms is the automatic segmentation of structures and substructures using a predefined atlas. But these algorithms have been limited to image volumes without gross abnormalities or pathologies and have thus been of limited use for applications such as the automatic segmentation of radiation sensitive structures for radiation therapy planning. The algorithm we present in this paper is an extension of a non-rigid registration algorithm we have previously developed (the Adaptive Basis Algorithm). This extension permits the use of the algorithm for the automatic segmentation of medical images even when structures have been displaced substantially. The algorithm automatically adjusts the stiffness of the transformation to permit larger displacements over regions that are known to be very compliant and smaller displacements over regions that are known to be less compliant. The stiffness map can be defined once and for all in an atlas. The algorithm has been tested and evaluated on head image volumes with large ventricular enlargements and head image volumes with large spaceoccupying lesions.
\end{abstract}

\section{INTRODUCTION}

Non-rigid inter-subject brain registration has been an important research topic during the last decade. A potential application, particularly useful in radiation therapy or neurosurgical planning, is the automatic delineation of brain structures and substructures using atlas-based methods. In this approach, structures of interest are labeled in a reference brain also called the atlas. The atlas is then registered to a subject's image volume. This establishes a point-to-point correspondence between the atlas and the patient's image volume. This, in turn, permits the transfer of labels from the atlas to the patient's volume. So far, methods proposed to solve this problem have been of limited use for patients with gross abnormalities or with extremely large ventricles.

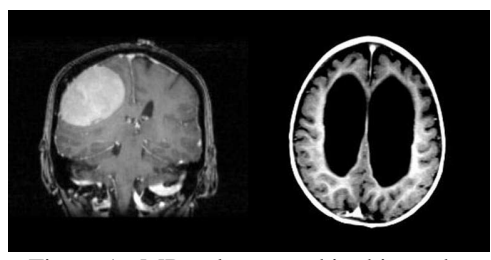

Figure 1: MR volume used in this study.

Figure 1 illustrates two examples of volumes used in this study. The left panel shows a large space-occupying lesion that has deformed its surrounding structures. The right panel shows one slice in a volume with enlarged ventricles. The challenges one faces when trying to register a normal volume to one of these are twofold: (1) the tumor does not exist in the atlas and (2) ventricular enlargement does not always happen by pushing and compressing surrounding structures. Often, cerebral matter simply disappears. Trying to compute a transformation that warps the atlas onto the patient volume may thus appear to be a futile exercise. But, as will be shown, the method we propose permits the accurate and automatic registration of an atlas with this type of image volume. We note that methods have been proposed earlier to register an atlas to volumes with space-occupying lesions (see for instance [1]). However, these require seeding the atlas with a pseudo-tumor and are thus not fully automatic.

\section{METHOD}

The method we propose is an extension of a non-rigid registration algorithm we have recently developed (Rohde et al. [2]). We modify this algorithm in two ways (1) we enforce the bijectivity of the transformations we compute and (2) we adapt the mechanical properties (i.e., the stiffness) of the transformation spatially.

\subsection{Bijectivity constraints}

Techniques have already been proposed to compute bijective transformations. In [3], Christensen et al. compute the forward and the backward transformations 
simultaneously and enforce consistency between these transformations. Thirion et al. [4] following the approach suggested by Burr et al. [5] compute the forward and the backward transformations independently but modify these transformations at each iteration to maintain their compatibility. It is the latest approach we have followed in this work.

\subsection{The automatic adjustment of the stiffness of the transformation}

Most of the techniques proposed so far for non-rigid registration uniformly impose constraints over the entire image domain. Notable exceptions can be found in Tanner et al. [6] in which control points in a B-spline based approach are coupled to locally enforce rigidity of the transformation, or in Little et al. [7] in which rigid structures are incorporated in a modified thin-plate spline registration algorithm. Both these techniques do, however, require segmentation of the rigid structures prior to registration and they enforce the rigidity constraints strictly. Registration methods based on physical models (see for instance Miga et al. [8] or Ferrant et al. [9]) do also, in principle, permit the adjustment of the mechanical properties of the transformation but they too require labeling of the various types of tissue and structures prior to registration.

In the Adaptive Basis Algorithm we use in this work, the displacement field that registers the images is modeled as a linear combination of radial basis function with compact support:

$$
p^{\prime}=p+\sum_{i=1, N} c_{i} \Phi_{i}\left(p_{i}-p\right)
$$

In which $p$ is a position vector in one image, $p^{\prime}$ the corresponding point in the other image and $\Phi_{i}$ are basis functions placed on an irregular grid at positions $p_{i}$. This algorithm also includes a constraint scheme that prevents the creation of physically incorrect transformations (i.e., transformations that would induce tearing or folding of the images). This scheme is implemented as a constraint between coefficients of adjacent basis functions.

At each iteration of the algorithm, the difference between the value of the coefficient being computed and of the coefficients of adjacent basis functions is evaluated and compared to an upper bound $\lambda$. When this limit is exceeded, the coefficient value is set to $\lambda$. This can be written in 1-D as:

$$
\left(c_{i+1}-c_{i}\right) \leq \lambda
$$

where $c_{i}$ and $c_{i+1}$ are adjacent basis functions coefficients.

Figure 2 that plots $p^{\prime}$ vs. $p$ illustrates this concept. The left panel shows a situation where all coefficients are the same. The right panel shows a situation where the difference between the coefficients of adjacent basis functions is large. The larger the difference between these coefficients the larger the local deformation generated by the transformation. This can be related to the mechanical properties of the transformation. The transformation shown on the left is stiffer or less compliant than the one shown on the right. Note also that the transformation $p^{\prime}=T(p)$ needs to remain monotonically increasing to avoid pixel cross-over. This is the case for the transformation shown on the left but not for the transformation shown on the right. Relaxing the constraint between basis function coefficients can thus increase the compliance of the transformation but also lead to non-physical transformations.
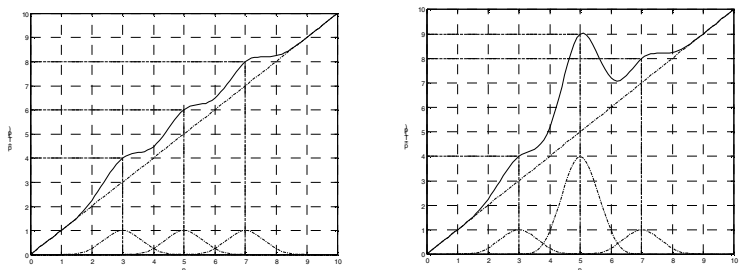

Figure 2: Left: $p^{\prime}=T(p)$ for a stiff transformation. Right: $p^{\prime}=T(p)$ for a very elastic transformation with pixel cross-over.

In our earlier work, the constraint between coefficients was used to guarantee the monotonicity of the transformation. Here, we use this constraint to adjust the mechanical properties of the transformation locally. Furthermore, because we are dealing with situations in which a one-to-one correspondence between an atlas and a particular image volume is not possible everywhere, we permit folding and tearing of the transformation over regions where it does not matter (e.g. within the ventricles) to preserve the physical properties of the transformation where it matters.

Because we want the algorithm to be automatic, labeling of regions that can deform more or less in each volume is not an option. But this can be done in the atlas because it requires labeling these regions only once. In the work presented here we associate an elasticity map with the atlas, which we call $\Lambda(p)$. So far, this map only contains two regions: the intra-ventricular region in which we use a high $\lambda_{\text {intra }}$ value, and the extra-ventricular region in which we use a low $\lambda_{\text {extra }}$ value. This choice of parameters allows the deformation to be more elastic over the ventricular regions that are typically compressed or expanded more than brain tissues. The information provided by the elasticity map is used in the registration process as follows:

$$
\left\{\begin{array}{l}
\lambda_{T_{A-2-S}^{n+1}}(p)=\Lambda\left(T_{A-2-S}^{n}(p)\right) \\
\lambda_{T_{S-2-A}}(p)=\Lambda(p)
\end{array}\right.
$$


in which $T_{A-2-S}$ is the transformation that registers the atlas to a subject image volume and $T_{S-2-A}$ is the transformation that registers the subject to the atlas. This equation shows that for a fixed location $p$ the elasticity map of the Atlas-to-Subject transformation is adjusted at each iteration. This is not required for the Subject-toAtlas transformation because the elasticity map is defined in the atlas.

The $\lambda$ values and the other registration parameters are experimentally optimized on representative images of a particular data set and then kept fixed for the registration of images of this data set.

\section{RESULTS}

\subsection{Synthetic images}

The performance of our local stiffness control approach was first evaluated on 2D binary images containing two similar circles or ellipses. The ellipses were generated from the circles by increasing the size of the vertical axis. The test consists in deforming circles into ellipses using different elasticity values for the right and left shape. Figures $3 \mathrm{a}$ and $3 \mathrm{~b}$ present results without and with the bijectivity constraint, respectively. In both cases, the top row presents results obtained with the same $\lambda$ value everywhere and the bottom row with a $\lambda$ value smaller for the right shape than for the left shape. In every case, the panel 1 shows a test image that has the same shape as the binary circle but that contains concentric circles with different intensity values to help in visualizing and comparing the transformations. These concentric circles were obtained with a distance map. The panel 2 shows the shape on the panel 1 deformed with the computed transformation. The panel 3 shows differences between the deformed binary circle and the target ellipse (common sections are showed in white, regions that do not correspond are shown in gray). The panel 4 shows the transformation applied to a regular grid and the panel 5 shows the difference between the forward (circle to ellipse) and the reverse (ellipse to circle) transformations.

Two main conclusions can be drawn from these figures. First, the bijectivity constraint we use produces forward and reverse transformations that are indeed inverse of each other. This, in turn, leads, to transformations that are more regular and that tend to be topologically correct. Second, the scheme we propose permits the local control of the transformation stiffness. Comparing the bottom and top panels in figures $3 \mathrm{a}$ and $3 \mathrm{~b}$, it is clear that by imposing a greater stiffness on the right circle, we have prevented the algorithm to deform it enough to cover the entire ellipse.

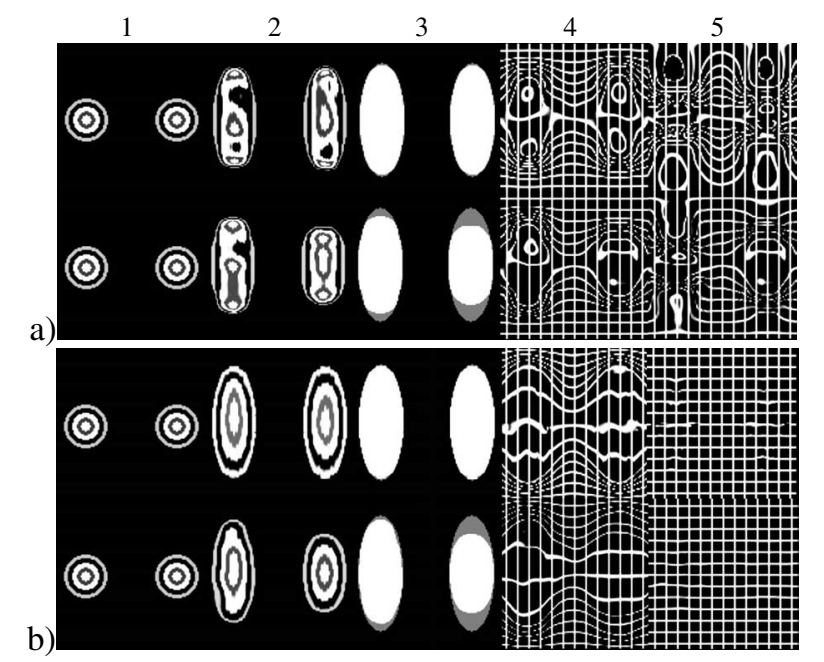

Figure 3: Circles deformed into ellipses with local control of stiffness. Rows: a) Without bijectivity. b) With bijectivity. Columns: 1. Source image. 2. Source deformed to the target. Up: with the same $\lambda$ value everywhere. Down: with a $\lambda$ value smaller for the right shape. 3 . Differences between the deformed binary circle and the target ellipse (common sections in white). 4. Transformation applied to a regular grid. 5. Difference between the forward and the reverse transformations.

\subsection{Real data}

Figure 4 and the top row of figure 5 show results obtained on one volume with enlarged ventricles and one with a large space-occupying lesion, respectively. In both cases, the left panel shows the atlas after an affine registration to its corresponding patient's volume (figure 1). The second and third panels show the registration results obtained with the same $\lambda$ value everywhere. To generate the results shown in the second panels, we used the $\lambda$ value we use for healthy brains. A larger value was used to generate the results shown on the third panels. The fourth panels show results obtained with a large value over the ventricular area and a normal one elsewhere. The corresponding deformation grids are shown below these images to help visualize and compare the smoothness and regularity of the transformations. Ventricles, tumor, and brain contours drawn on the patient's volumes are copied on the deformed atlas and grids.

The bottom row of figure 5 shows a surface rendering of the deformed ventricles and brainstem. These were obtained by deforming the structures defined in the atlas with the computed transformations. The tumor was segmented by hand and displayed to show its position relative to the brainstem and ventricles and the effect it has on these.

The second panels in figure 4 and 5 clearly show that a $\lambda$ value used for non-pathological brains leads to a transformation that is too stiff to completely expand or contract the ventricles. The third panels show that 
increasing the value of $\lambda$ compresses/expands the ventricles satisfactorily but also lead to a transformation that is irregular and, at places, non-physical. The right panels show that a local control of the stiffness of the transformation is a good compromise between these two extremes. The transformation is smooth and does not produce folding of the images over regions of interest while allowing a large deformation and compression of the ventricles. The monotonicity of the transformation is not preserved where the images differ topologically (e. g. inside the tumor) but these are regions where the correspondence one to one doesn't matter.

In the large tumor volume, the tumor not only compresses the ventricles but it also compresses the top of the brainstem. Comparing the two rightmost bottom panels of figure 5 , one observes that a uniformly large $\lambda$ value leads to a transformation that flattens the top of the brainstem but also changes its shape far away from the tumor. Using two different $\lambda$ values leads to a transformation that flattens the top of the brainstem but preserves its shape further down.

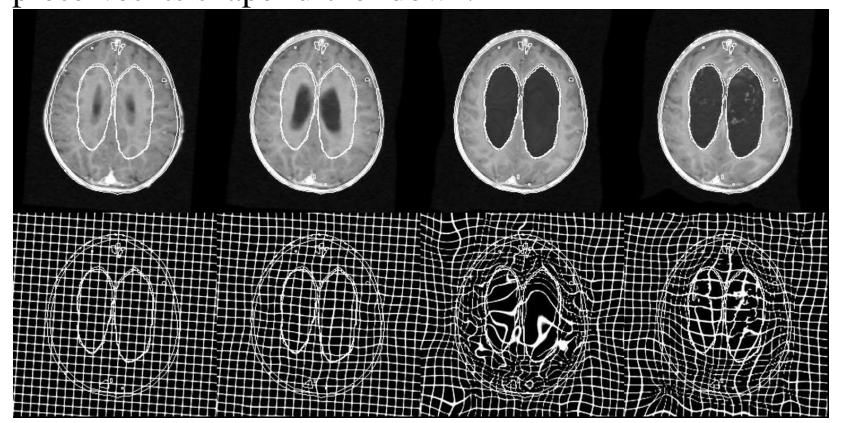

Figure 4: Registration of a child brain with enlarged ventricles.

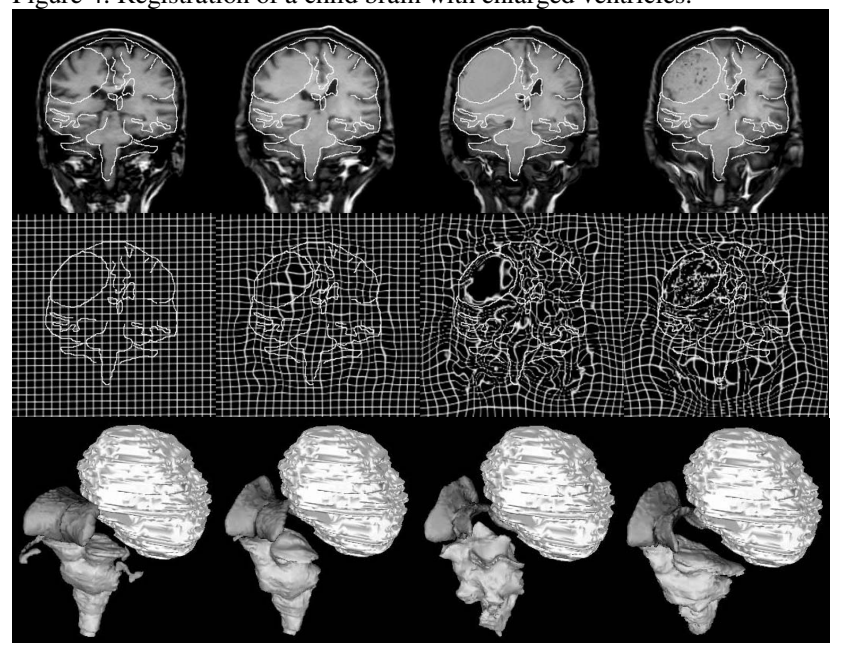

Figure 5: Registration and segmentation of an adult brain with a large space-occupying tumor.

\section{CONCLUSION}

The results presented in this paper indicate that local control of the stiffness of the transformation represents a property particularly useful for automatic registration of medical images with large abnormalities or pathologies. The method proposed here is fully automatic. It doesn't require any previous segmentation of the patient's volume nor seeding of the atlas and the elasticity map used is defined only once by the user in the atlas. For the cases used in this study, we defined areas in the volume (the lateral ventricles) that can deform more than the rest of the image. Futher work includes using our scheme to constrain the deformation of rigid structures such the vertebras or semi-rigid structures such as the mid-sagital plane. The validation of the results presented in this paper was performed visually and qualitatively. Future work includes validating our algorithm quantitatively and comparing to existing methods such as the one described in [1].

\section{REFERENCES}

[1] M. Bach Cuadra, J. Gomez, P. Hagmann, C. Pollo, J. Villemure, B. M. Dawant, and J.-P. Thiran, "Atlas-based Segmentation of Pathological Brains using a Model of Tumor Growth," in LNCS: Proceedings of MICCAI'02, vol. 16, 2002, pp. 380-387.

[2] G. K. Rohde, A. Aldroubi, and B. M. Dawant, "The Adaptive Bases Algorithm for Intensity-Based Nonrigid Image Registration," in IEEE Transactions on Medical Imaging, Volume: 22, Issue: 11, 2003, pp. 1470- 1479.

[3] G. E. Christensen and H. J. Johnson, "Consistent Image Registration," vol. 20, no. 7, 2001, pp. 568-582.

[4] J. P. Thirion, "Image matching as a diffusion process: an analogy with Maxwell's demons," in Medical Image Analysis, vol. 2, no. 3, 1998, pp. 243-260.

[5] D. J. Burr, "A dynamic model for image registration," in Computer Graphics and Image Processing, vol. 15, 1981, pp. $102-112$.

[6] C. Tanner, A. Degenhard, J. A. Schnabel, A. D. Castellano Smith, C. Hayes, L. I. Sonoda, M. O. Leach, D. R. Leach, D. R. Hose, D. L. G. Hill, and D. J. Hawkes, "Volume and shape preservation of enhancing lesions when applying nonrigid registration to a time series of contrast enhancing MR breast images," in LNCS: Proceedings of MICCAI'00, vol. 2489, 2000, pp. 327-337.

[7] J. A. Little, D. L. G. Hill, and D. J. Hawkes, "Deformations Incorporating Rigid Structures," in Comp. Vis. Image Understand., vol. 66, 1997, pp. 223-232.

[8] M.I. Miga, K. D. Paulsen, F. E. Kennedy, P. J. Hoopes, A. Hartov, D.W. Roberts, "Initial In-Vivo Analysis of 3d Heterogeneous Brain Computations for Model-Updated Image-Guided Neurosurgery," in LNCS: Proceedings of MICCAI'98, vol. 1496, 1998, pp. 743-752.

[9] M. Ferrant, A. Nabavi, B. Macq, P.M. Black, F. A. Jolesz, R. Kikinis, S. K. Warfield, "Serial Registration of Intraoperative MR Images of the Brain," Medical Image Analysis 6, 2002, pp. 337-359.

\section{ACKNOWLEDGEMENTS}

This work was supported, in parts, by grant NIH R21 CA89657$01 \mathrm{~A} 2$. 